\title{
Arthroscopic ankle fusion only has a limited advantage over the open operation if the condition is similar: a retrospective comparative study
}

\author{
Chenggong Wang \\ Xiangya Hospital Central South University \\ Can Xu \\ Xiangya Hospital Central South University \\ Mingqing Li \\ Xiangya Hospital Central South University \\ Hui Li \\ Xiangya Hospital Central South University \\ Long Wang \\ Xiangya Hospital Central South University \\ Da Zhong \\ Xiangya Hospital Central South University \\ Hua Liu ( $\square$ dr_liuhua_xyyy@126.com ) \\ Xiangya Hospital Central South University https://orcid.org/0000-0003-2034-6407
}

Research article

Keywords: Arthroscopic ankle fusion, Open ankle fusion, Selection bias, Follow-up, Questionnaire survey

Posted Date: January 7th, 2020

DOI: https://doi.org/10.21203/rs.2.20212/v1

License: (c) (1) This work is licensed under a Creative Commons Attribution 4.0 International License. Read Full License 


\section{Abstract}

Background: A great deal of research suggests that arthroscopic ankle fusion (AAF) has advantages over open ankle fusion (OAF), but these outcomes would be imprecise because of a selection bias. The purpose of this study is to verify which is better for ankle fusion, AAF or OAF. We regrouped the OAF group into two subgroups according to whether the disease condition is similar to AAF group. The goal is to minimize the impact of disease severity, thereby reducing selection bias to some extent.

Methods: We retrospectively analyzed the data of ankle fusion in our hospital between July 2015 and October 2018. 43 patients were enrolled and divided into AAF group ( $n=17)$ and OAF group ( $n=26)$. In order to eliminate selection bias, we divided OAF group into serious condition subgroup (SC subgroup) $(n=15)$ and not serious condition subgroup (NSC subgroup) $(n=11)$. The NSC subgroup condition is similar to AAF group. Then, we compared the differences between these groups. All patients were followed up at least one year after operation. We analyzed data, including etiology composition, surgical time, intra-op blood loss, reduction of albumin, total hospital stays, union time, fusion situation, complications, radiological examination, functional score and questionnaire survey. Then we performed statistical analyses.

Results: We found that the etiological components of AAF group and OAF group were different, the etiological components of AAF group and NSC subgroup were similar. We found that AAF group has advantages over OAF group and SC subgroup in general. However, except in terms of surgical trauma, hospital stays and short-term complications occurred, the AAF group has not obvious advantages over NSC subgroup, including intra-op blood loss, fusion condition, postoperative function score and postoperative patient satisfaction; and AAF group need more surgical time than the NSC subgroup.

Conclusions: The arthroscopic ankle fusion can bring a good curative effect. However, if the condition is similar, the arthroscopic ankle fusion only has a limited advantage over the open operation in beautiful incision, perioperative soft tissue protection and enhanced recovery after surgery.

\section{Background}

Ankle fusion is a reliable and effective option in the treatment of end-stage ankle arthritis and pain ${ }^{[1]}$. While ankle fusion carries a high rate of union, the optimal surgical method continues to be debated with more than 40 techniques described in the literature ${ }^{[2-6]}$. Although most open operation methods have achieved a good curative effect, they have however been associated with many complications ${ }^{[7,8]}$. These complications of open ankle fusion resulted in the development of less invasive techniques such as arthroscopic ankle fusion ${ }^{[9]}$. A great deal of research suggests that arthroscopic ankle fusion has advantages over open operation such as faster time to union, lower morbidity, lower blood loss, faster rehabilitation and shorter hospital stay [10-14]. Many scholars believe that the reason of these advantages by arthroscopic ankle fusion probably because periosteal stripping is not necessary, and the local circulation remains intact, creating a more favourable environment for fusion to occur ${ }^{[15]}$.

However, most of these studies are case series report, only a few of them are prospective controlled studies. In fact, there are a number of etiologies for the patients need ankle fusion, and the disease conditions are difference for the patients. The different etiologies and disease conditions will affect the choice of operation method. In other words, it is very likely that some severe patients with poor curative effect cannot choose arthroscopic ankle fusion method but only open method, and the comparison results are also likely to favor arthroscopic ankle fusion method. But such outcomes of the comparative study would be imprecise because of a grouping selection bias ${ }^{[16]}$. In fact, such selection bias can hardly be avoided in the study of the surgical effect of ankle fusion.

In this study, we regrouped the open surgery group into serious condition subgroup and not serious condition subgroup, the latter condition is similar to arthroscopic ankle fusion group. Then, we compared the differences between these groups. The goal is to minimize the impact of disease severity, thereby reducing selection bias to some extent. It's worth noting that some different results seemed to be found unlike most studies that have been done.

\section{Method}

\section{Study design}

This study has been approved by the Ethics Committee of Xiangya Hospital (No.201907007). We retrospectively analyzed a series of data of patients with ankle fusion of our department between July 2015 and October 2018. Data were obtained from the registration system of foot and ankle surgery department, the medical record information system and the follow-up system of patients' service center of Xiangya Hospital. All patients included in this retrospective study were all agreed to participate in the study and have been signed written consent. All cases were operated by professor Liu.H, who has more than 50 surgical experience of open ankle fusion and more than 30 surgical experience of arthroscopic ankle fusion. The inclusion criteria were as follows: 1) primary ankle fusion surgery; 2 ) unilateral ankle fusion; 3 ) voluntary provision of medical records to this study. The exclusion criteria were as follows: 1) refused to participate in this study; 2) ankle fusion by using external fixator; 3 ) the homolateral lower limb includes other operations, such as joint arthroplasty, internal fixation or other joint fusion; 4) physical activity disorders caused by other disease, such as stroke; 5) mental illness.

By carefully searching the system with our study design, 43 patients were enrolled in our study and received final follow-up (Table 1). We divided the patients into two groups by surgical method: arthroscopic ankle fusion group (AAF group) ( $n=17$ ) (Fig. 1A, 1B); and open ankle fusion group (OAF group) $(n=26)$. The purpose of this study was to analyze the effect of surgical methods on ankle fusion only. In order to eliminate selection bias, we divided OAF group into two subgroups, which were used for more detailed comparison with AAF group. The OAF group was divided into serious condition subgroup (SC subgroup) ( $n=15)$ and not serious condition subgroup (NSC subgroup) $(n=11)$. The condition of SC subgroup was relatively complicated (Fig. $2 A$, 2B); 
during the operation of SC subgroup, osteotomy, orthopedics, large lesion cleaning or large bone graft reconstruction were required; none of these surgical procedures above could be performed through arthroscopic surgery. However, the condition in NSC subgroup was relatively simple (Fig. 3A, 3B), almost all cases in NSC subgroup could be chosen arthroscopic fusion except for a few cases, which could not establish an arthroscopic surgical pathway because of excessive hyperplasia around the joint or excessive narrowing of the joint space. Basic information of the patients is presented in Table 1.

Table 1

Patients demographics and preoperative conditions.

\begin{tabular}{|c|c|c|c|c|c|c|c|c|c|c|c|c|}
\hline & \multicolumn{3}{|c|}{$\begin{array}{l}\text { Between-group difference } \\
\text { (all patients) }\end{array}$} & \multicolumn{3}{|c|}{$\begin{array}{l}\text { Difference detailed into } \\
\text { subgroup } \\
\text { (not similar condition) }\end{array}$} & \multicolumn{3}{|c|}{$\begin{array}{l}\text { Difference detailed into } \\
\text { subgroup } \\
\text { (similar condition) }\end{array}$} & \multicolumn{3}{|c|}{$\begin{array}{l}\text { Internal difference } \\
\text { (OAF, not similar } \\
\text { condition) }\end{array}$} \\
\hline & AAF & OAF & $\mathbf{P}$ & AAF & Sc & $\mathbf{P}$ & AAF & NSC & $\mathbf{P}$ & SC & NSC & $\mathbf{P}$ \\
\hline Number of patients & 17 & 26 & & 17 & 15 & & 17 & 11 & & 15 & 11 & \\
\hline $\begin{array}{l}\text { Mean of Age (years) } \\
(95 \% \mathrm{Cl})\end{array}$ & $\begin{array}{l}54.76 \pm \\
14.11\end{array}$ & $\begin{array}{l}55.35 \pm \\
12.52\end{array}$ & NS & $\begin{array}{l}54.76 \pm \\
14.11\end{array}$ & $\begin{array}{l}53.33 \pm \\
14.82\end{array}$ & NS & $\begin{array}{l}54.76 \pm \\
14.11\end{array}$ & $\begin{array}{l}58.09 \pm \\
8.35\end{array}$ & NS & $\begin{array}{l}53.33 \\
\pm 14.82\end{array}$ & $\begin{array}{l}58.09 \\
\pm 8.35\end{array}$ & NS \\
\hline $\begin{array}{l}\text { Gender } \\
\text { (male/Female) }\end{array}$ & $10 / 7$ & $16 / 10$ & NS & $10 / 7$ & $11 / 4$ & NS & $10 / 7$ & $7 / 4$ & NS & $11 / 4$ & $7 / 4$ & NS \\
\hline Smoker (No. and \%) & $\begin{array}{l}3 \\
(17.6 \%)\end{array}$ & $\begin{array}{l}7 \\
(26.9 \%)\end{array}$ & NS & $\begin{array}{l}3 \\
(17.6 \%)\end{array}$ & $\begin{array}{l}4 \\
(26.7 \%)\end{array}$ & NS & $\begin{array}{l}3 \\
(17.6 \%)\end{array}$ & $\begin{array}{l}3 \\
(27.3 \%)\end{array}$ & NS & $\begin{array}{l}4 \\
(26.7 \%)\end{array}$ & $\begin{array}{l}3 \\
(27.3 \%)\end{array}$ & NS \\
\hline Diabetes (No. and \%) & $\begin{array}{l}4 \\
(23.5 \%)\end{array}$ & $\begin{array}{l}3 \\
(11.5 \%)\end{array}$ & NS & $\begin{array}{l}4 \\
(25.3 \%)\end{array}$ & $1(6.7 \%)$ & NS & $\begin{array}{l}4 \\
(23.5 \%)\end{array}$ & 2 & NS & $\begin{array}{l}1 \\
(6.7 \%)\end{array}$ & $\stackrel{2}{(18.2 \%)}$ & NS \\
\hline Mean of BMI & $\begin{array}{l}26.55 \pm \\
5.23\end{array}$ & $\begin{array}{l}28.93 \pm \\
5.95\end{array}$ & NS & $\begin{array}{l}26.55 \pm \\
5.23\end{array}$ & $\begin{array}{l}29.21 \pm \\
5.22\end{array}$ & NS & $\begin{array}{l}26.55 \pm \\
5.23\end{array}$ & $\begin{array}{l}28.55 \pm \\
7.09\end{array}$ & NS & $\begin{array}{l}29.21 \\
\pm 5.22\end{array}$ & $\begin{array}{l}28.55 \\
\pm 7.09\end{array}$ & NS \\
\hline $\begin{array}{l}\text { Mean of AOFAS score } \\
\text { (Preoperative) }\end{array}$ & $\begin{array}{l}36.2 \pm \\
13.5\end{array}$ & $\begin{array}{l}32.5 \pm \\
11.8\end{array}$ & NS & $\begin{array}{l}36.2 \pm \\
13.5\end{array}$ & $\begin{array}{l}30.7 \pm \\
12.8\end{array}$ & NS & $\begin{array}{l}36.2 \pm \\
13.5\end{array}$ & $\begin{array}{l}34.8 \pm \\
10.5\end{array}$ & NS & $\begin{array}{l}30.7 \pm \\
12.8\end{array}$ & $\begin{array}{l}34.8 \pm \\
10.5\end{array}$ & NS \\
\hline $\begin{array}{l}\text { Mean of follow-up } \\
\text { time (months) }\end{array}$ & $\begin{array}{l}31.94 \pm \\
11.07\end{array}$ & $\begin{array}{l}34.81 \pm \\
9.41\end{array}$ & NS & $\begin{array}{l}31.94 \pm \\
11.07\end{array}$ & $\begin{array}{l}36.53 \pm \\
8.49\end{array}$ & NS & $\begin{array}{l}31.94 \pm \\
11.07\end{array}$ & $\begin{array}{l}32.45 \pm \\
10.47\end{array}$ & NS & $\begin{array}{l}36.53 \\
\pm 8.49\end{array}$ & $\begin{array}{l}32.45 \\
\pm 10.47\end{array}$ & NS \\
\hline
\end{tabular}

All patients were followed up 3 months, 6 months and 1 year after surgery, and accepted at least one follow-up after postoperative one year again. Mean follow-up time: 33.7 months (range: 14 months to 49 months)

\section{Operative Technique}

\section{Arthroscopic ankle fusion group}

The operations were performed under sciatic and femoral nerve block anesthesia with additional general anesthesia. All patients were used two surgical entries located in front of the ankle space, medial to tibialis anterior tendon and lateral to peroneus tertius tendon. A small number of patients have added posterior ankle entries on either side of the achilles tendon if necessary. We used arthroscopic burrs and soft-tissue shavers to remove synovium, cartilage and osteonecrotic areas. After that, we made the subchondral bone to bleed heavily. The medial and lateral gutter joint surfaces were also denuded. We fused the ankle to a plantar flexion of $90^{\circ}$, ectropion of $5^{\circ}$, external of $5^{\circ}$, and make sure the ankle axis in an ideal position. We checked the position by anteroposterior and lateral intraoperative fluoroscopic images and direct vision until we are satisfied. According to the specific bone defect of joint space, we used pressure bone grafting by using excess osteophytes, proximal tibia bone graft (PTBG) or demineralized bone matrix (DBM). Fixation was achieved with two or three large cannulated screws $(6.5 \mathrm{~mm}, 6.9 \mathrm{~mm}$ and $7.2 \mathrm{~mm})$ under fluoroscopic guidance (Fig. 1). The first screw entered from the lateral fibula or lateral tibial about 15-25 mm above the ankle line and penetrated the collum tali to the caput tali. The second screw entered from the medial tibia about 15-25 mm above the ankle line and penetrated to the area close to subtalar joint of the lateral talus. If we needed to install the third screw, its entry point was 5-10 mm higher than the second screw in general and penetrated to the area close to subtalar joint of the lateral talus too. We ensured the adequate screw fixation and compression across the fusion site under intraoperative fluoroscopic images and direct physical examination. We closed the wounds with simple interrupted nonabsorbable monofilament sutures.

\section{Open ankle fusion group}

In SC subgroup ( $n=15)$, a transfibular approach was used in the majority of patients (13/15) (Fig. 2). In the transfibular approach, we made a curvilinear incision over the lateral ankle and removed the distal fibula. For majority of patients $(n=9)$, the distal fibula was separated or crushed, for impacted or structural bone grafting; and for other patients $(n=4)$, the distal fibula was split into two half portions longitudinally, the medial portion was used for bone grafting, and the lateral portion was retained for reconstruction by using screws. Then we exposed tibiotalar joint and remove synovium, cartilage and osteonecrotic areas. After that, we made the subchondral bone to bleed heavily with a $1.5-2.0 \mathrm{~mm}$ drill. The ankle was then provisionally pinned in position after the medial gutter joint surfaces were also denuded. We fused the ankle to a plantar flexion of $90^{\circ}$, ectropion of $5^{\circ}$, external of $5^{\circ}$, and make sure the ankle axis in an ideal position. In most instances $(n=9)$, we placed one or two guidewires for cannulated screws $(6.5 \mathrm{~mm}, 6.9 \mathrm{~mm}$ and $7.2 \mathrm{~mm})$. Then we 
installed the cannulated screws, the first screw entered from the area close to subtalar joint of the lateral talus and penetrated to the medial tibia about $15-$ $25 \mathrm{~mm}$ above the ankle line, or the installation trajectory was just the opposite. If we needed to install the second screw, its entry point was located at the lateral tibia about 15-25 mm above the ankle line and penetrated the collum tali to the caput tali. We placed a locking plate laterally with three or four 3.5$\mathrm{mm}$ screws placed into the talus and three or four screws placed proximally into the distal tibia. For other patients $(n=4)$, the procedures for joint cleaning and bone grafting were the same as above, but the tibial talus joint was fused by using two or three large cannulated screws (6.5 mm, $6.9 \mathrm{~mm}$ and $7.2 \mathrm{~mm}$ ) similar to AAF group; finally, the lateral portion of distal fibula mentioned above was secured to the lateral malleolus by two 3.5-mm screws. The remaining patients underwent either an anterior $(n=2)$ approach to use previous incisions. In this approach, we did not remove the distal fibula after the ankle articular cavity was exposed.

In NSC subgroup $(n=11)$, patients were treated with an anterior $(n=5)$, bilateral anterior-oblique $(n=4)$, anteromedial $(n=1)$ or anterolateral $(n=1)$ approach. In these approaches, we did not remove the distal fibula too. The joint was cleaned and fixed temporarily to the above position. The ankles were fixed by using two $(n=5)$ or three $(n=6)$ cannulated screws similar to the AAF group. (Fig. 3$)$

\section{Postoperative Management}

After surgery, the patients of two groups were required to raise the limb, and the use of antibiotics was necessary. For OAF group, drainage was usually postoperatively. For both two groups, the patient wore a plaster slab until the stitch of wound was removed immediately after surgery, and then wore a below-knee protective plaster cast. Patients were encouraged to mobilize non-weight bearing for the first 4-6 weeks, partially weight bearing for several weeks according to the doctor's advice, and full weight bearing thereafter.

\section{Measurement and follow-up}

We carefully searched and collected the following data through the medical records and follow-up system: Surgical time, intra-op blood loss, reduction of albumin (ALB, the difference of albumin value between preoperative 2 days and postoperative 2 days), total hospital stays, union time, fusion situation and complications; the American Orthopedic Foot and Ankle Society (AOFAS) ankle and hindfoot score ${ }^{[17,18]}$ and the outcomes of follow-up questionnaire which referred to Musculoskeletal Outcomes Data Evaluation and Management Scale (MODEMS) questionnaire ${ }^{[19]}$. We got these data through outpatient or visiting services.

\section{Statistical analysis}

After all the results of each time point of follow-up have been collected, we made statistical analyses of the data: Independent Samples t-test was used to assess the difference of the age, BMI, follow-up time, AOFAS score, surgical time, intra-op blood loss, reduction of ALB, total hospital stays, union time and questionnaire outcomes. Chi-squared test was used to analyze the difference of the gender, smoker, diabetes and etiology. Statistical analyses were performed using SPSS 20.0 software (SPSS Inc., Chicago, IL, USA). P values less than 0.05 were considered statistically significant.

\section{Results}

\section{Etiology and composition}

By analyzing the etiological components of each group, we found that the etiological components of AAF group and NSC subgroup were similar, mainly including osteoarthritis, posttrauma arthritis and infectious arthritis, NSC group was treated rheumatoid arthritis and urarthritis patients; the difference was that the etiology of SC group included talus necrosis, clubfoot and charcot arthritis. The number and proportion of osteoarthritis $(7$ cases, $41.2 \%)(P=$ 0.008 ) and posttrauma arthritis cause by ligament lesions ( 5 cases, $29.4 \%)(P=0.018)$ of AAF group was significantly more than the OAF group ( 2 cases, $7.7 \%$; 1 cases, 3.8\%); the number and proportion of talus necrosis of AAF group ( 0 case) was significantly less than the OAF group ( 4 cases, $15.4 \%)(P=$ 0.038). Similarly, the number and proportion of osteoarthritis (7 cases, $41.2 \%)(P=0.005)$ and posttrauma arthritis cause by ligament lesions $(5$ cases, 29.4\%) ( $P=0.022$ ) of AAF group was significantly more than the SC subgroup ( 0 case; 0 case); the number and proportion of talus necrosis of AAF group ( 0 case) was significantly less than the SC subgroup (4 cases, 26.7\%) $(\mathrm{P}=0.023)$. The number and proportion of talus necrosis of SC subgroup (4 cases, $26.7 \%$ ) was significantly more than the NSC subgroup ( 0 case $)(P=0.026)$. (Table 2$)$ 
Table 2

Patients etiology conditions.

\begin{tabular}{|c|c|c|c|c|c|c|c|c|c|c|c|c|}
\hline & \multicolumn{3}{|c|}{$\begin{array}{l}\text { Between-group difference } \\
\text { (all patients) }\end{array}$} & \multicolumn{3}{|c|}{$\begin{array}{l}\text { Difference detailed into } \\
\text { subgroup } \\
\text { (not similar condition) }\end{array}$} & \multicolumn{3}{|c|}{$\begin{array}{l}\text { Difference detailed into } \\
\text { subgroup } \\
\text { (similar condition) }\end{array}$} & \multicolumn{3}{|c|}{$\begin{array}{l}\text { Internal difference } \\
\text { (OAF, not similar condition) }\end{array}$} \\
\hline & $\begin{array}{l}\operatorname{AAF}(n \\
=17)\end{array}$ & $\begin{array}{l}\text { OAF(n } \\
=26)\end{array}$ & $\mathbf{P}$ & $\begin{array}{l}\text { AAF(n } \\
=17)\end{array}$ & $\begin{array}{l}\operatorname{SC}(n= \\
15)\end{array}$ & $\mathbf{P}$ & $\begin{array}{l}\text { AAF(n } \\
=17)\end{array}$ & $\begin{array}{l}\text { NSC(n } \\
=11)\end{array}$ & & $\begin{array}{l}\operatorname{sc}(n= \\
15)\end{array}$ & $\begin{array}{l}\text { NSC(n } \\
=11)\end{array}$ & $\mathbf{P}$ \\
\hline \multicolumn{13}{|l|}{$\begin{array}{l}\text { Etiology (No. and } \\
\% \text { ) }\end{array}$} \\
\hline Osteoarthritis & $\begin{array}{l}7 \\
(41.2 \%)\end{array}$ & $\begin{array}{l}2 \\
(7.7 \%)\end{array}$ & 0.008 & $\begin{array}{l}7 \\
(41.2 \%)\end{array}$ & 0 & 0.005 & $\begin{array}{l}7 \\
(41.2 \%)\end{array}$ & 2 & NS & 0 & 2 & NS \\
\hline $\begin{array}{l}\text { Posttrauma } \\
\text { arthritis } \\
\text { (fracture) }\end{array}$ & $\begin{array}{l}3 \\
(17.6 \%)\end{array}$ & $\begin{array}{l}11 \\
(42.3 \%)\end{array}$ & NS & $\begin{array}{l}3 \\
(17.6 \%)\end{array}$ & $\begin{array}{l}7 \\
(46.7 \%)\end{array}$ & NS & $\begin{array}{l}3 \\
(17.6 \%)\end{array}$ & $\begin{array}{l}4 \\
(36.4 \%)\end{array}$ & NS & $\begin{array}{l}7 \\
(46.7 \%)\end{array}$ & $\begin{array}{l}4 \\
(36.4 \%)\end{array}$ & NS \\
\hline $\begin{array}{l}\text { Posttrauma } \\
\text { arthritis } \\
\text { (ligament } \\
\text { lesions) }\end{array}$ & $\begin{array}{l}5 \\
(29.4 \%)\end{array}$ & $\begin{array}{l}1 \\
(3.8 \%)\end{array}$ & 0.018 & $\begin{array}{l}5 \\
(29.4 \%)\end{array}$ & 0 & 0.022 & $\begin{array}{l}5 \\
(29.4 \%)\end{array}$ & $\begin{array}{l}1 \\
(9.1 \%)\end{array}$ & NS & 0 & $\begin{array}{l}1 \\
(9.1 \%)\end{array}$ & NS \\
\hline $\begin{array}{l}\text { Infectious } \\
\text { arthritis }\end{array}$ & $\begin{array}{l}2 \\
(11.8 \%)\end{array}$ & $\begin{array}{l}2 \\
(7.7 \%)\end{array}$ & NS & 2 & $\begin{array}{l}1 \\
(6.7 \%)\end{array}$ & NS & $\begin{array}{l}2 \\
(11.8 \%)\end{array}$ & $\begin{array}{l}1 \\
(9.1 \%)\end{array}$ & NS & $\begin{array}{l}1 \\
(6.7 \%)\end{array}$ & $\begin{array}{l}1 \\
(9.1 \%)\end{array}$ & NS \\
\hline $\begin{array}{l}\text { Rheumatoid } \\
\text { arthritis }\end{array}$ & 0 & $\begin{array}{l}2 \\
(7.7 \%)\end{array}$ & NS & 0 & 0 & NS & 0 & $\begin{array}{l}2 \\
(18.2 \%)\end{array}$ & NS & 0 & $\begin{array}{l}2 \\
(18.2 \%)\end{array}$ & NS \\
\hline Urarthritis & 0 & $\begin{array}{l}1 \\
(3.8 \%)\end{array}$ & NS & 0 & 0 & NS & 0 & $\begin{array}{l}1 \\
(9.1 \%)\end{array}$ & NS & 0 & $\begin{array}{l}1 \\
(9.1 \%)\end{array}$ & NS \\
\hline $\begin{array}{l}\text { Talus } \\
\text { necrosis }\end{array}$ & 0 & $\begin{array}{l}4 \\
(15.4 \%)\end{array}$ & 0.038 & 0 & $\begin{array}{l}4 \\
(26.7 \%)\end{array}$ & 0.023 & 0 & 0 & NS & $\begin{array}{l}4 \\
(26.7 \%)\end{array}$ & 0 & 0.026 \\
\hline Clubfoot & 0 & $\begin{array}{l}2 \\
(7.7 \%)\end{array}$ & NS & 0 & $\begin{array}{l}2 \\
(13.3 \%)\end{array}$ & NS & 0 & 0 & NS & $\begin{array}{l}2 \\
(13.3 \%)\end{array}$ & 0 & NS \\
\hline $\begin{array}{l}\text { Charcot } \\
\text { arthritis }\end{array}$ & 0 & $\begin{array}{l}1 \\
(3.8 \%)\end{array}$ & NS & 0 & $\begin{array}{l}1 \\
(6.7 \%)\end{array}$ & NS & 0 & 0 & NS & $\begin{array}{l}1 \\
(6.7 \%)\end{array}$ & 0 & NS \\
\hline
\end{tabular}

AAF: arthroscopic ankle fusion group; OAF: open ankle fusion group (demographic composition: OAF = SC + NSC); SC: serious condition subgroup; NSC: not serious condition subgroup. P: P values: $a=0.05$, (Chi-squared test)

\section{Operation outcomes}

Through postoperative imaging examination, all groups can be found the joint space were filled and pressurized. (Fig. 1-3) The surgical time of NSC subgroup (123.6 $\pm 18.6 \mathrm{~min})$ was significantly less than the AAF group $(140.5 \pm 22.2 \mathrm{~min})(P=0.046)$ and $\mathrm{SC}$ subgroup $(149.3 \pm 23.1 \mathrm{~min})(P=0.006)$. The intra-op blood loss of AAF group $(137.1 \pm 49.7 \mathrm{ml})$ was significantly less than the OAF group (184.6 $\pm 62.9 \mathrm{ml})(P=0.012)$ and the SC subgroup (206.7 \pm $67.8 \mathrm{ml})(\mathrm{P}=0.002)$, and the intra-op blood loss of NSC subgroup $(154.5 \pm 41.6 \mathrm{ml})$ was significantly less than the SC subgroup $(206.7 \pm 67.8 \mathrm{ml})(\mathrm{P}=$ 0.034). The reduction of ALB (the difference of albumin value between preoperative 2 days and postoperative 2 days) (1.28 $\pm 0.28 \mathrm{~g} / \mathrm{L}$ ) of $\mathrm{AAF}$ group was significantly less than the OAF group $(3.35 \pm 1.19 \mathrm{~g} / \mathrm{L})(P=0.000)$, the $\mathrm{SC}$ subgroup $(3.49 \pm 1.22 \mathrm{~g} / \mathrm{L})(\mathrm{P}=0.000)$ and the NSC subgroup $(3.15 \pm 1.19 \mathrm{~g} / \mathrm{L})(\mathrm{P}$ $=0.000)$. Similarly, the total hospital stays of AAF group (6.3 \pm 2.5 days) was significantly less than the OAF group $(11.0 \pm 2.7$ days $)(P=0.000)$, the $S C$ subgroup (11.4 \pm 3.4 days) $(P=0.000)$ and the NSC subgroup $(10.5 \pm 1.5$ days $)(P=0.000)$. One patient of AAF group, two patients of SC subgroup and one patient of NSC subgroup occurred transient paralysis of nerve after operation; three patients of SC subgroup and one patient of NSC subgroup occurred delayed wound healing after operation; and one patient of SC subgroup occurred soft tissue infection after operation. (Table 3, Fig. 4) 
Table 3

Outcomes.

\begin{tabular}{|c|c|c|c|c|c|c|c|c|c|c|c|c|}
\hline & \multicolumn{3}{|c|}{$\begin{array}{l}\text { Between-group difference } \\
\text { (all patients) }\end{array}$} & \multicolumn{3}{|c|}{$\begin{array}{l}\text { Difference detailed into } \\
\text { subgroup } \\
\text { (not similar condition) }\end{array}$} & \multicolumn{3}{|c|}{$\begin{array}{l}\text { Difference detailed into } \\
\text { subgroup } \\
\text { (similar condition) }\end{array}$} & \multicolumn{3}{|c|}{$\begin{array}{l}\text { Intemal difference } \\
\text { (OAF, not similar condition) }\end{array}$} \\
\hline & $\begin{array}{l}\text { AAF(n } \\
=17)\end{array}$ & $\begin{array}{l}\text { OAF(n } \\
=26)\end{array}$ & $\mathbf{P}$ & $\begin{array}{l}\text { AAF(n } \\
=17)\end{array}$ & $\begin{array}{l}\operatorname{sc}(n= \\
15)\end{array}$ & $\mathbf{P}$ & $\begin{array}{l}\text { AAF(n } \\
=17)\end{array}$ & $\begin{array}{l}\text { NSC(n } \\
=11)\end{array}$ & $\mathbf{P}$ & $\begin{array}{l}\operatorname{sc}(n= \\
15)\end{array}$ & $\begin{array}{l}\text { NSC(n } \\
=11)\end{array}$ & $\mathbf{P}$ \\
\hline $\begin{array}{l}\text { Mean of } \\
\text { surgical time } \\
\text { (min.) }\end{array}$ & $\begin{array}{l}140.5 \\
\pm 22.2\end{array}$ & $\begin{array}{l}138.5 \pm \\
24.6\end{array}$ & NS & $\begin{array}{l}140.5 \\
\pm 22.2\end{array}$ & $\begin{array}{l}149.3 \pm \\
23.1\end{array}$ & NS & $\begin{array}{l}140.5 \\
\pm 22.2\end{array}$ & $\begin{array}{l}123.6 \\
\pm 18.6\end{array}$ & 0.046 & $\begin{array}{l}149.3 \pm \\
23.1\end{array}$ & $\begin{array}{l}123.6 \\
\pm 18.6\end{array}$ & 0.006 \\
\hline $\begin{array}{l}\text { Mean of intra- } \\
\text { op blood loss } \\
(\mathrm{ml})\end{array}$ & $\begin{array}{l}137.1 \\
\pm 49.7\end{array}$ & $\begin{array}{l}184.6 \pm \\
62.9\end{array}$ & 0.012 & $\begin{array}{l}137.1 \\
\pm 49.7\end{array}$ & $\begin{array}{l}206.7 \pm \\
67.8\end{array}$ & 0.002 & $\begin{array}{l}137.1 \\
\pm 49.7\end{array}$ & $\begin{array}{l}154.5 \\
\pm 41.6\end{array}$ & NS & $\begin{array}{l}206.7 \pm \\
67.8\end{array}$ & $\begin{array}{l}154.5 \\
\pm 41.6\end{array}$ & 0.034 \\
\hline $\begin{array}{l}\text { Mean of } \\
\text { reduction of } \\
\text { ALB } *(g / L)\end{array}$ & $\begin{array}{l}1.28 \pm \\
0.28\end{array}$ & $\begin{array}{l}3.35 \pm \\
1.19\end{array}$ & 0.000 & $\begin{array}{l}1.28 \pm \\
0.28\end{array}$ & $\begin{array}{l}3.49 \pm \\
1.22\end{array}$ & 0.000 & $\begin{array}{l}1.28 \pm \\
0.28\end{array}$ & $\begin{array}{l}3.15 \pm \\
1.19\end{array}$ & 0.000 & $\begin{array}{l}3.49 \pm \\
1.22\end{array}$ & $\begin{array}{l}3.15 \pm \\
1.19\end{array}$ & NS \\
\hline $\begin{array}{l}\text { Mean of total } \\
\text { hospital stays } \\
\text { (days) }\end{array}$ & $\begin{array}{l}6.3 \pm \\
2.5\end{array}$ & $\begin{array}{l}11.0 \pm \\
2.7\end{array}$ & 0.000 & $\begin{array}{l}6.3 \pm \\
2.5\end{array}$ & $\begin{array}{l}11.4 \pm \\
3.4\end{array}$ & 0.000 & $\begin{array}{l}6.3 \pm \\
2.5\end{array}$ & $\begin{array}{l}10.5 \pm \\
1.5\end{array}$ & 0.000 & $\begin{array}{l}11.4 \pm \\
3.4\end{array}$ & $\begin{array}{l}10.5 \pm \\
1.5\end{array}$ & NS \\
\hline $\begin{array}{l}\text { Mean of union } \\
\text { time (weeks) }\end{array}$ & $\begin{array}{l}12.4 \pm \\
1.9\end{array}$ & $\begin{array}{l}14.6 \pm \\
3.4\end{array}$ & 0.019 & $\begin{array}{l}12.4 \pm \\
1.9\end{array}$ & $\begin{array}{l}15.5 \pm \\
3.5\end{array}$ & 0.003 & $\begin{array}{l}12.4 \pm \\
1.9\end{array}$ & $\begin{array}{l}12.8 \pm \\
2.3\end{array}$ & NS & $\begin{array}{l}15.5 \pm \\
3.5\end{array}$ & $\begin{array}{l}12.8 \pm \\
2.3\end{array}$ & 0.035 \\
\hline $\begin{array}{l}\text { No fusion (No. } \\
\text { and \%) }\end{array}$ & 0 & $2(7.7 \%)$ & & 0 & $2(13.3 \%)$ & & 0 & 0 & & $2(13.3 \%)$ & 0 & \\
\hline $\begin{array}{l}\text { Complications } \\
\text { situation }\end{array}$ & \multicolumn{12}{|c|}{$\begin{array}{l}\text { AAF group: transient paralysis of nerve ( } 1 \text { case) } \\
\text { SC subgroup: delayed wound healing ( } 3 \text { cases); transient paralysis of nerve }(2 \text { cases); infection ( } 1 \text { case) } \\
\text { NSC subgroup: delayed wound healing ( } 1 \text { case); transient paralysis of nerve ( } 1 \text { case) }\end{array}$} \\
\hline
\end{tabular}

\section{Follow-up outcomes}

At 3 months after the operation, all patients were followed up in the outpatient and completed the AOFAS score. At 6 months and 1 year after the operation, there were respectively three and four patients lost to follow-up, but the proportion in each group was less than $15 \%$. At the final follow-up, all patients were followed up and completed the AOFAS score and questionnaire survey. (Table 4) 
Table 4

Outcomes of AOFAS score.

\begin{tabular}{|c|c|c|c|c|c|c|c|c|c|c|c|c|}
\hline & \multicolumn{3}{|c|}{$\begin{array}{l}\text { Between-group difference } \\
\text { (all patients) }\end{array}$} & \multicolumn{3}{|c|}{$\begin{array}{l}\text { Difference detailed into } \\
\text { subgroup } \\
\text { (not similar condition) }\end{array}$} & \multicolumn{3}{|c|}{$\begin{array}{l}\text { Difference detailed into } \\
\text { subgroup } \\
\text { (similar condition) }\end{array}$} & \multicolumn{3}{|c|}{$\begin{array}{l}\text { Internal difference } \\
\text { (OAF, not similar } \\
\text { condition) }\end{array}$} \\
\hline & AAF & OAF & $\mathbf{P}$ & AAF & SC & $\mathbf{P}$ & AAF & NSC & $\mathbf{P}$ & SC & NSC & $\mathbf{P}$ \\
\hline \multicolumn{13}{|l|}{ Mean of AOFAS score } \\
\hline $\begin{array}{l}\text { Within } 2 \text { weeks } \\
\text { before surgery }\end{array}$ & $\begin{array}{l}36.2 \pm \\
13.5 \\
(n= \\
17)\end{array}$ & $\begin{array}{l}32.5 \pm \\
11.8 \\
(n= \\
26)\end{array}$ & NS & $\begin{array}{l}36.2 \pm \\
13.5 \\
(n= \\
17)\end{array}$ & $\begin{array}{l}30.7 \pm \\
12.8 \\
(n= \\
15)\end{array}$ & NS & $\begin{array}{l}36.2 \pm \\
13.5 \\
(n= \\
17)\end{array}$ & $\begin{array}{l}34.8 \pm \\
10.5 \\
(n= \\
11)\end{array}$ & NS & $\begin{array}{l}30.7 \\
\pm \\
12.8 \\
(\mathrm{n}= \\
15)\end{array}$ & $\begin{array}{l}34.8 \\
\pm \\
10.5 \\
(\mathrm{n}= \\
11)\end{array}$ & NS \\
\hline Post-op 3 months & $\begin{array}{l}63.1 \pm \\
3.3 \\
(n= \\
17)\end{array}$ & $\begin{array}{l}59.6 \pm \\
4.7 \\
(n= \\
26)\end{array}$ & 0.013 & $\begin{array}{l}63.1 \pm \\
3.3 \\
(n= \\
17)\end{array}$ & $\begin{array}{l}57.9 \pm \\
4.7 \\
(n= \\
15)\end{array}$ & 0.001 & $\begin{array}{l}63.1 \pm \\
3.3 \\
(n= \\
17)\end{array}$ & $\begin{array}{l}61.9 \pm \\
3.9 \\
(n= \\
11)\end{array}$ & NS & $\begin{array}{l}57.9 \\
\pm 4.7 \\
(\mathrm{n}= \\
15)\end{array}$ & $\begin{array}{l}61.9 \\
\pm 3.9 \\
(\mathrm{n}= \\
11)\end{array}$ & 0.031 \\
\hline Post-op 6 months & $\begin{array}{l}70.5 \pm \\
5.7 \\
(n= \\
15)\end{array}$ & $\begin{array}{l}66.7 \pm \\
5.1 \\
(n= \\
25)\end{array}$ & 0.034 & $\begin{array}{l}70.5 \pm \\
5.7 \\
(n= \\
15)\end{array}$ & $\begin{array}{l}64.9 \pm \\
3.8 \\
(n= \\
15)\end{array}$ & 0.004 & $\begin{array}{l}70.5 \pm \\
5.7 \\
(n= \\
15)\end{array}$ & $\begin{array}{l}69.4 \pm \\
5.7 \\
(n= \\
10)\end{array}$ & NS & $\begin{array}{l}64.9 \\
\pm 3.8 \\
(\mathrm{n}= \\
15)\end{array}$ & $\begin{array}{l}69.4 \\
\pm 5.7 \\
(\mathrm{n}= \\
10)\end{array}$ & 0.027 \\
\hline Post-op 1 year & $\begin{array}{l}76.2 \pm \\
3.9 \\
(n= \\
16)\end{array}$ & $\begin{array}{l}73.0 \pm \\
7.1 \\
(n= \\
23)\end{array}$ & NS & $\begin{array}{l}76.2 \pm \\
3.9 \\
(n= \\
16)\end{array}$ & $\begin{array}{l}72.1 \pm \\
6.2 \\
(n= \\
14)\end{array}$ & 0.037 & $\begin{array}{l}76.2 \pm \\
3.9 \\
(n= \\
16)\end{array}$ & $\begin{array}{l}74.3 \pm \\
8.1 \\
(n=9)\end{array}$ & NS & $\begin{array}{l}72.1 \\
\pm 6.2 \\
(n= \\
14)\end{array}$ & $\begin{array}{l}74.3 \\
\pm 8.1 \\
(n= \\
9)\end{array}$ & NS \\
\hline $\begin{array}{l}\text { Final (at least } 1 \text { year } \\
\text { post-op) }\end{array}$ & $\begin{array}{l}77.7 \pm \\
3.8 \\
(n= \\
17)\end{array}$ & $\begin{array}{l}75.8 \pm \\
4.5 \\
(n= \\
26)\end{array}$ & NS & $\begin{array}{l}77.7 \pm \\
3.8 \\
(n= \\
17)\end{array}$ & $\begin{array}{l}75.4 \pm \\
3.7 \\
(n= \\
15)\end{array}$ & NS & $\begin{array}{l}77.7 \pm \\
3.8 \\
(n= \\
17)\end{array}$ & $\begin{array}{l}76.3 \pm \\
5.6 \\
(n= \\
11)\end{array}$ & NS & $\begin{array}{l}75.4 \\
\pm 3.7 \\
(n= \\
15)\end{array}$ & $\begin{array}{l}76.3 \\
\pm 5.6 \\
(\mathrm{n}= \\
11)\end{array}$ & NS \\
\hline
\end{tabular}

\section{Radiological and fusion examination}

At 3 months after operation, no obvious internal fixation loosening were observed in all groups; for most of the AAF group and NSC subgroup patients, we can found a lot of bone bridges in the joint space, and the ankle fusion were ideal; but there were only a part of patients in SC subgroup can be found ideal ankle fusion. (Fig. 1-3) The union time of AAF group (12.4 \pm 1.9 weeks) was significantly less than the OAF group (14.6 \pm 3.4 weeks) (P=0.019) and the SC subgroup (15.5 \pm 3.5 weeks) $(P=0.003)$, and the union time of NSC subgroup (12.8 \pm 2.3 weeks) was significantly less than the SC subgroup (15.5 \pm 3.5 weeks) $(P=0.035)$. In SC subgroup, there were two cases occurred nonfusion after primary ankle fusion surgical; both of them underwent refusion revision surgery. (Table 3, Fig. 4)

\section{Functional score}

By analyzing the results of AOFAS scores, the curative effect and recovery rate of AAF group and NSC subgroup were both better than the SC subgroup after operation until one year after operation: The postoperative 3 months AOFAS score of AAF group (63.1 \pm 3.3$)$ was significantly better than the OAF group (59.6 \pm 4.7$)(P=0.013)$ and the SC subgroup $(57.9 \pm 4.7)(P=0.001)$, and the postoperative 3 months AOFAS score of NSC subgroup (61.9 \pm 3.9$)$ was significantly better than the SC subgroup $(57.9 \pm 4.7)(P=0.031)$. The postoperative 6 months AOFAS score of AAF group (70.5 \pm 5.7$)$ was significantly better than the OAF group $(66.7 \pm 5.1)(P=0.034)$ and the SC subgroup $(64.9 \pm 3.8)(P=0.004)$, and the postoperative 6 months AOFAS score of NSC subgroup (69.4 \pm 5.7$)$ was significantly better than the SC subgroup $(64.9 \pm 3.8)(P=0.027)$. The postoperative 1 year AOFAS score of AAF group (76.2 \pm 3.9$)$ was significantly better than the SC subgroup $(72.1 \pm 6.2)(P=0.037)$. But there was no significant difference between these three groups from one year after operation, and the AOFAS score mean of each group was greater than 75 points. (Table 4, Fig. 5)

\section{Questionnaire survey}

The results of questionnaire survey (Fig. 6) shows: First, no matter which groups were not satisfied with the improvement of the pain and other symptoms through question A-1. Second, each patient gave a high score to question A-2 and A-3, which suggested that almost all patients can do some mellow work without walking long-distance, and can sleep well. Third, when asked about participating in usual job and recreational activities, almost all the patients of AAF group and NSC subgroup gave significantly better answers than the SC subgroup. Four, overall, the patients were felt somewhat satisfied if they had to spend the rest of their life with the symptoms they have at the final follow-up of the study.

\section{Discussion}


Although there were many studies considered the arthroscopic ankle fusion achieve similar or better rates of fusion and better follow-up outcomes than open fusion. However, most of these studies are case series report, only a few of them are prospective controlled studies. In this study, the open surgery group, which was regrouped according to the difference severity of disease, was supposed to reduce grouping selection bias. It's worth noting that some different results seemed to be found, and we discuss them as follows.

\section{About etiology}

By statistical analysis, we found that the etiological components of AAF group and OAF group were different, the etiological components of AAF group and NSC subgroup were similar, mainly including the cases not need osteotomy, orthopedics, large lesion cleaning or large bone graft reconstruction, such as osteoarthritis, posttrauma arthritis cause by ligament lesions and rheumatoid arthritis ${ }^{[20,21]}$. However, the etiological components of AAF group and SC subgroup were significantly different, such as advanced talus necrosis, severe clubfoot and charcot arthritis, which surgical process was relatively complicated and the surgical effect was poor ${ }^{[22-24]}$. This also supports the correctness of the grouping of subgroup for OAF group to some extent. We believed that such experimental design of further grouping can improve the credibility. After all, roughly dividing patients into AAF group and OAF group according to surgical methods would lead to selection bias, especially for retrospective studies ${ }^{[25,26]}$. Therefore, this study compared the AAF group with the SC subgroup and the NSC subgroup respectively; it would easier to exclude the selection bias caused by the severity of the disease, so as to objectively analyze the differences caused by surgical methods.

\section{About operation}

Through postoperative imaging examination, all groups can be found the joint space were filled and pressurized, it means that the arthroscopic ankle fusion technology can deal with the joint surface well when the disease condition is similar to NSC subgroup. The surgical time of NSC subgroup was the fastest compared with other groups; it means that AAF group has no advantage in surgical time compared with OAF group, because the clearance of joint surface is very complicated and slow by arthroscopic technology. AAF group and NSC subgroup have obvious advantages in intra-op blood loss compared with SC subgroup; the possible reason is that the increase in surgical time cancels out the advantage of fewer traumas compared with NSC subgroup. However, it's worth noting that AAF group has obvious advantages in avoid of ALB reduction, total hospital stays and avoid of complications compared with other groups; the reason can be explained by the small trauma of AAF group. Some studies considered that arthroscopic ankle fusion can result in lower complication rate and shorter hospital stay as compared to open-ankle fusions ${ }^{[27,28]}$.

\section{About radiological and fusion}

At 3 months after operation, for most of the AAF group and NSC subgroup patients, we can found a lot of bone bridges in the joint space, and the ankle fusion were ideal; but there were only a part of patients in SC subgroup can be found ideal ankle fusion. The union time of AAF group and NSC subgroup were significantly less than the OAF group and the SC subgroup. In SC subgroup, there were two cases occurred nonfusion after primary ankle fusion surgical; both of them underwent refusion revision surgery. This shows that the fusion time of AAF group which similar to that of NSC subgroup is faster than SC subgroup until one year after the surgery, but has no advantage in long-term follow-up. The possible reason is the surgical trauma and illness condition of SC subgroup are more severe. There are many similar studies, it's reported that the overall non-union rates after anterior arthroscopic assisted ankle fusions are $8.6 \%{ }^{[29]}$; and some researches shows the minimally invasive operation would have good fusion under the premise the cannulated screws

are installed well ${ }^{[30,31]}$. However, none of these studies took into account the selection bias of grouping caused by the severity of the disease, so it cannot be generally considered that the fusion condition of arthroscopic technology has an advantage over open technology.

\section{About functional score}

By analyzing the results of AOFAS scores, the curative effect and recovery rate of AAF group and NSC subgroup were both better than the SC subgroup after operation until one year after operation; but there was no significant difference between these three groups from one year after operation, and the AOFAS score mean of each group was greater than 75 points, which can be considered that the function is good according to the current mainstream view. This shows that the scores of all groups failed to achieve excellent, the reason is the nature of ankle surgery itself. The gait of patients had been still showed slight limp after ankle fusion, and the patients would appeared pain and swelling when they walk for a long time, which has been widely reported ${ }^{\text {[32-35] }}$ Ebalard et al. reported that $84 \%$ of patients complained of pain after a minimum follow-up of 10 years ${ }^{[36]}$. In other studies, the prevalence of osteoarthrosis ranged from $24-100 \%$ in the subtalar joint and from $18-77 \%$ in the Chopart joints ${ }^{[37]}$. What's more notable is that the recovery condition of AAF group which similar to that of NSC subgroup is faster than SC subgroup until one year after the surgery, but has no advantage in long-term follow-up. The possible reason is the surgical trauma and illness condition of SC subgroup are more severe, so it cannot be generally considered that the function score of arthroscopic technology is better than open technology. 


\section{About questionnaire survey}

Patient expectation and satisfaction may be valuable measures for defining the success of an operative intervention [38, 39] , so we designed a part of questionnaire survey in our study. The expectation scores used in the present study were referred to the MODEMS scores, which are currently the only scores that are applicable to ankle outcomes ${ }^{[19]}$.

In the part of questionnaire survey, through question A-1 we can see no matter which groups were not satisfied with the improvement of the pain and other symptoms, the possible reason was the gait of patients had been still showed slight limp after ankle fusion, and the patients would appeared pain and swelling when they walk for a long time, which lead a bad subjective feeling of the patients. Notably, dissatisfaction with symptoms appeared to be higher in the AAF group, possibly because these patients had a higher expectation because they thought the minimally invasive surgery would help them got a faster recovery. Each patient gave a high score to question A-2 and A-3, which suggested that almost all patients can do some mellow work without walking long-distance, and can sleep well; but it is worth noting that there are 3 individuals chose N/A in question A-3, which reflected the diseases need ankle fusion have little effects on sleep even before operation. When asked about participating in usual job and recreational activities, almost all the patients of AAF group and NSC subgroup gave significantly better answers than the SC subgroup; the possible reason should be that the patients in the SC subgroup thought it was difficult to return to usual job and recreational activities due to the severe disease, poor surgical effect and fair subjective effect. Finally, almost all the patients felt somewhat satisfied if they had to spend the rest of their life with the symptoms they have at the final follow-up of the study; this result shows that although ankle fusion cannot make the patients obtain excellent functions, it can still help the patients in all groups got recovery to some extent and make them satisfied. Even more remarkable, question A-2 and A-3 revealed some differences that were not demonstrated by AOFAS score; the possible reason is the patient's expectations may therefore be quite different from the actual result of function in many case, it is also worth noting that this discrepancy is a potential source of patient dissatisfaction ${ }^{[40,41]}$.

\section{Limitation}

Firstly, the follow-up time of the study is still not long enough to observe the complications such as degeneration of the surrounding joint. Secondly, it is necessary to research the survival rate of ankle joint in each group after surgery on the basis of long-term observation in the future. Thirdly, this study is only a retrospective study. A prospective study which excluding confounding factors by good experimental design should be conducted, even randomized controlled trial.

\section{Conclusions}

In summary, we found that AAF group has advantages over OAF group in surgical procedure, fusion condition and postoperative recovery in general. However, after the selection bias was minimized from the grouping of OAF group again, which was divided into two subgroups according to disease condition, we found that: AAF group only has advantages over SC subgroup, which had a severe disease and could only use open surgery and often required large lesion clearance and reconstruction. It's worth noting that except in terms of surgical trauma, hospital stays and short-term complications occurred, the AAF group has not obvious advantages over NSC subgroup, which disease condition and operation method are similar to AAF group, including intra-op blood loss, fusion condition, postoperative function score and postoperative patient satisfaction; and AAF group need more surgical time than the NSC subgroup. Therefore, we believe that the arthroscopic ankle fusion can bring a good curative effect; however, if the condition is similar, the arthroscopic ankle fusion only has a limited advantage over the traditional open operation in beautiful incision, perioperative soft tissue protection and enhanced recovery after surgery.

\section{List Of Abbreviations}

AAF: arthroscopic ankle fusion; OAF: open ankle fusion; SC: serious condition; NSC: not serious condition; BMI: body mass index; ALB: albumin; PTBG: proximal tibia bone graft; DBM: demineralized bone matrix; AOFAS: American Orthopedic Foot and Ankle Society; MODEMS: Musculoskeletal Outcomes Data Evaluation and Management Scale.

\section{Declarations}

\section{Acknowledgements}

This study was strongly supported by the Ethics Committee of Xiangya Hospital, the registration system of foot and ankle surgery department, the medical record information system and the follow-up system of patients' service center of Xiangya Hospital.

\section{Funding}

This study was funded by Natural Science Foundation of Hunan Province (2016JJ4108). 
The datasets used and/or analyzed during the study are available from the corresponding author upon reasonable request.

\section{Authors' contributions}

Liu.H and Wang.CG contributed to the investigation, methodology, data curation, and preparation of the original draft. Wang.CG contributed to the formal analysis, data curation, and preparation of the original draft. Xu.C contributed to the data curation and preparation of the original draft. Wang.C and Li.MQ prepared the original draft. Xu.C and Li.H performed data validation. Liu.H and Xu.C contributed to the surgical guidance. Li.MQ edited and reviewed the manuscript. All authors read and approved the final manuscript.

\section{Ethics approval and consent to participate}

This study was approved by the medical ethics committee of the Xiangya Hospital of Centre South University (IRB (C) No. 201907007). All procedures performed in studies were in accordance with the ethical standards of our institutional ethical committee. Informed consent was obtained from all individual participants included in the study.

\section{Consent for publication}

All patients involved had given informed consent.

\section{Competing interests}

The authors declare that they have no competing interests.

\section{References}

[1] Mitchell PM, Douleh DG, Thomson AB. Comparison of Ankle Fusion Rates With and Without Anterior Plate Augmentation. Foot Ankle Int. 2017. 38(4): 419-423.

[2] Gharehdaghi M, Rahimi H, Mousavian A. Anterior ankle arthrodesis with molded plate: technique and outcomes. Arch Bone Jt Surg. 2014 . 2(3): 203-9.

[3] Hayes BJ, Gonzalez T, Smith JT, Chiodo CP, Bluman EM. Ankle Arthritis: You Can't Always Replace It. J Am Acad Orthop Surg. 2016. 24(2): e29-38.

[4] Haddad SL, Coetzee JC, Estok R, Fahrbach K, Banel D, Nalysnyk L. Intermediate and long-term outcomes of total ankle arthroplasty and ankle arthrodesis. A systematic review of the literature. J Bone Joint Surg Am. 2007. 89(9): 1899-905.

[5] Plaass C, Knupp M, Barg A, Hintermann B. Anterior double plating for rigid fixation of isolated tibiotalar arthrodesis. Foot Ankle Int. 2009. 30(7): 631-9.

[6] Mohamedean A, Said HG, El-Sharkawi M, El-Adly W, Said GZ. Technique and short-term results of ankle arthrodesis using anterior plating. Int Orthop. 2010. 34(6): 833-7.

[7] Thordarson DB. Fusion in posttraumatic foot and ankle reconstruction. J Am Acad Orthop Surg. 2004. 12(5): 322-33.

[8] Lawton CD, Butler BA, Dekker RG 2nd, Prescott A, Kadakia AR. Total ankle arthroplasty versus ankle arthrodesis-a comparison of outcomes over the last decade. J Orthop Surg Res. 2017. 12(1): 76.

[9] Jain SK, Tiernan D, Kearns SR. Analysis of risk factors for failure of arthroscopic ankle fusion in a series of 52 ankles. Foot Ankle Surg. 2016. 22(2): 91 6.

[10] Veljkovic AN, Daniels TR, Glazebrook MA, et al. Outcomes of Total Ankle Replacement, Arthroscopic Ankle Arthrodesis, and Open Ankle Arthrodesis for Isolated Non-Deformed End-Stage Ankle Arthritis. J Bone Joint Surg Am. 2019. 101(17): 1523-1529.

[11] Woo BJ, Lai MC, Ng S, Rikhraj IS, Koo K. Clinical outcomes comparing arthroscopic vs open ankle arthrodesis. Foot Ankle Surg. 2019 .

[12] Quayle J, Shafafy R, Khan MA, Ghosh K, Sakellariou A, Gougoulias N. Arthroscopic versus open ankle arthrodesis. Foot Ankle Surg. 2018. 24(2): 137142.

[13] Dannawi Z, Nawabi DH, Patel A, Leong JJ, Moore DJ. Arthroscopic ankle arthrodesis: are results reproducible irrespective of pre-operative deformity. Foot Ankle Surg. 2011. 17(4): 294-9. 
[14] Duan X, Yang L, Yin L. Arthroscopic arthrodesis for ankle arthritis without bone graft. J Orthop Surg Res. 2016. $11(1): 154$.

[15] Hendrickx R, de Leeuw P, Golano P, van Dijk CN, Kerkhoffs G. Safety and efficiency of posterior arthroscopic ankle arthrodesis. Knee Surg Sports Traumatol Arthrosc. 2015. 23(8): 2420-2426.

[16] Elston DM. Selection bias. J Am Acad Dermatol. 2019.

[17] Kitaoka HB, Alexander IJ, Adelaar RS, et al. Clinical Rating Systems for the Ankle-Hindfoot, Midfoot, Hallux, and Lesser Toes. Foot Ankle Int. 1997. 18(3): 187-188

[18] Ibrahim T, Beiri A, Azzabi M, Best AJ, Taylor GJ, Menon DK. Reliability and validity of the subjective component of the American Orthopaedic Foot and Ankle Society clinical rating scales. J Foot Ankle Surg. 2007. 46(2): 65-74.

[19] Zywiel MG, Mahomed A, Gandhi R, Perruccio AV, Mahomed NN. Measuring expectations in orthopaedic surgery: a systematic review. Clin Orthop Relat Res. 2013. 471(11): 3446-56.

[20] Xu C, Zhang MY, Lei GH, et al. Biomechanical evaluation of tenodesis reconstruction in ankle with deltoid ligament deficiency: a finite element analysis. Knee Surg Sports Traumatol Arthrosc. 2012. 20(9): 1854-62.

[21] Chen $\mathrm{C}, \mathrm{Lu} \mathrm{H}, \mathrm{Hu} \mathrm{J}$, et al. Anatomic reconstruction of anterior talofibular ligament with tibial tuberosity-patellar tendon autograft for chronic lateral ankle instability. J Orthop Surg (Hong Kong). 2018. 26(2): 2309499018780874.

[22] Goebel M, Gerdesmeyer L, Mückley T, et al. Retrograde intramedullary nailing in tibiotalocalcaneal arthrodesis: a short-term, prospective study. J Foot Ankle Surg. 2006. 45(2): 98-106.

[23] Devries JG, Philbin TM, Hyer CF. Retrograde intramedullary nail arthrodesis for avascular necrosis of the talus. Foot Ankle Int. 2010. 31(11): 965-72.

[24] Millett PJ, O'Malley MJ, Tolo ET, Gallina J, Fealy S, Helfet DL. Tibiotalocalcaneal fusion with a retrograde intramedullary nail: clinical and functional outcomes. Am J Orthop (Belle Mead NJ). 2002. 31(9): 531-6.

[25] Barrett J, Zaltz A, Geary M, Sermer M, Kingdom J. Beware selection bias. CMAJ. 2017. 189(34): E1096.

[26] Hernán MA, Hernández-Díaz S, Robins JM. A structural approach to selection bias. Epidemiology. 2004. 15(5): 615-25.

[27] Peterson KS, Lee MS, Buddecke DE. Arthroscopic versus open ankle arthrodesis: a retrospective cost analysis. J Foot Ankle Surg. 2010. 49 (3): 242-7.

[28] Townshend D, Di Silvestro M, Krause F, et al. Arthroscopic versus open ankle arthrodesis: a multicenter comparative case series. J Bone Joint Surg Am. 2013. 95(2): 98-102.

[29] Abicht BP, Roukis TS. Incidence of nonunion after isolated arthroscopic ankle arthrodesis. Arthroscopy. 2013. 29(5): 949-54.

[30] Kamijo S, Kumai T, Tanaka S, Mano T, Tanaka Y. Comparison of compressive forces caused by various cannulated cancellous screws used in arthroscopic ankle arthrodesis. J Orthop Surg Res. 2017. 12(1): 7.

[31] Zhu M, Yuan CS, Jin ZM, et al. Initial stability and stress distribution of ankle arthroscopic arthrodesis with three kinds of 2-screw configuration fixation: a finite element analysis. J Orthop Surg Res. 2018. 13(1): 263.

[32] Papa JA, Myerson MS. Pantalar and tibiotalocalcaneal arthrodesis for post-traumatic osteoarthrosis of the ankle and hindfoot. J Bone Joint Surg Am. 1992. 74(7): 1042-9.

[33] Wang S, Huang Z, Xiong G, Chen G, Yin Z, Jiang H. Modified Blair ankle fusion for ankle arthritis. Chin J Traumatol. 2014. 17(3): 136-40.

[34] Kendal AR, Cooke P, Sharp R. Arthroscopic ankle fusion for avascular necrosis of the talus. Foot Ankle Int. 2015. 36(5): 591-7.

[35] de Leeuw PA, Hendrickx RP, van Dijk CN, Stufkens SS, Kerkhoffs GM. Midterm results of posterior arthroscopic ankle fusion. Knee Surg Sports Traumatol Arthrosc. 2016. 24(4): 1326-31.

[36] Ebalard M, Le Henaff G, Sigonney G, et al. Risk of osteoarthritis secondary to partial or total arthrodesis of the subtalar and midtarsal joints after a minimum follow-up of 10 years. Orthop Traumatol Surg Res. 2014. 100(4 Suppl): S231-7.

[37] Ling JS, Smyth NA, Fraser EJ, et al. Investigating the relationship between ankle arthrodesis and adjacent-joint arthritis in the hindfoot: a systematic review. J Bone Joint Surg Am. 2015. 97(6): 513-20.

[38] Younger AS, Wing KJ, Glazebrook M, et al. Patient expectation and satisfaction as measures of operative outcome in end-stage ankle arthritis: a prospective cohort study of total ankle replacement versus ankle fusion. Foot Ankle Int. 2015. 36(2): 123-34. 
[39] Mancuso CA, Graziano S, Briskie LM, et al. Randomized trials to modify patients' preoperative expectations of hip and knee arthroplasties. Clin Orthop Relat Res. 2008. 466(2): 424-31.

[40] Ghomrawi HM, Franco Ferrando N, Mandl LA, Do H, Noor N, Gonzalez Della Valle A. How Often are Patient and Surgeon Recovery Expectations for Total Joint Arthroplasty Aligned? Results of a Pilot Study. HSS J. 2011. 7(3): 229-34.

[41] Ghomrawi HM, Mancuso CA, Westrich GH, Marx RG, Mushlin Al, Expectations Discordance Study Group. Discordance in TKA expectations between patients and surgeons. Clin Orthop Relat Res. 2013. 471(1): 175-80.

\section{Figures}

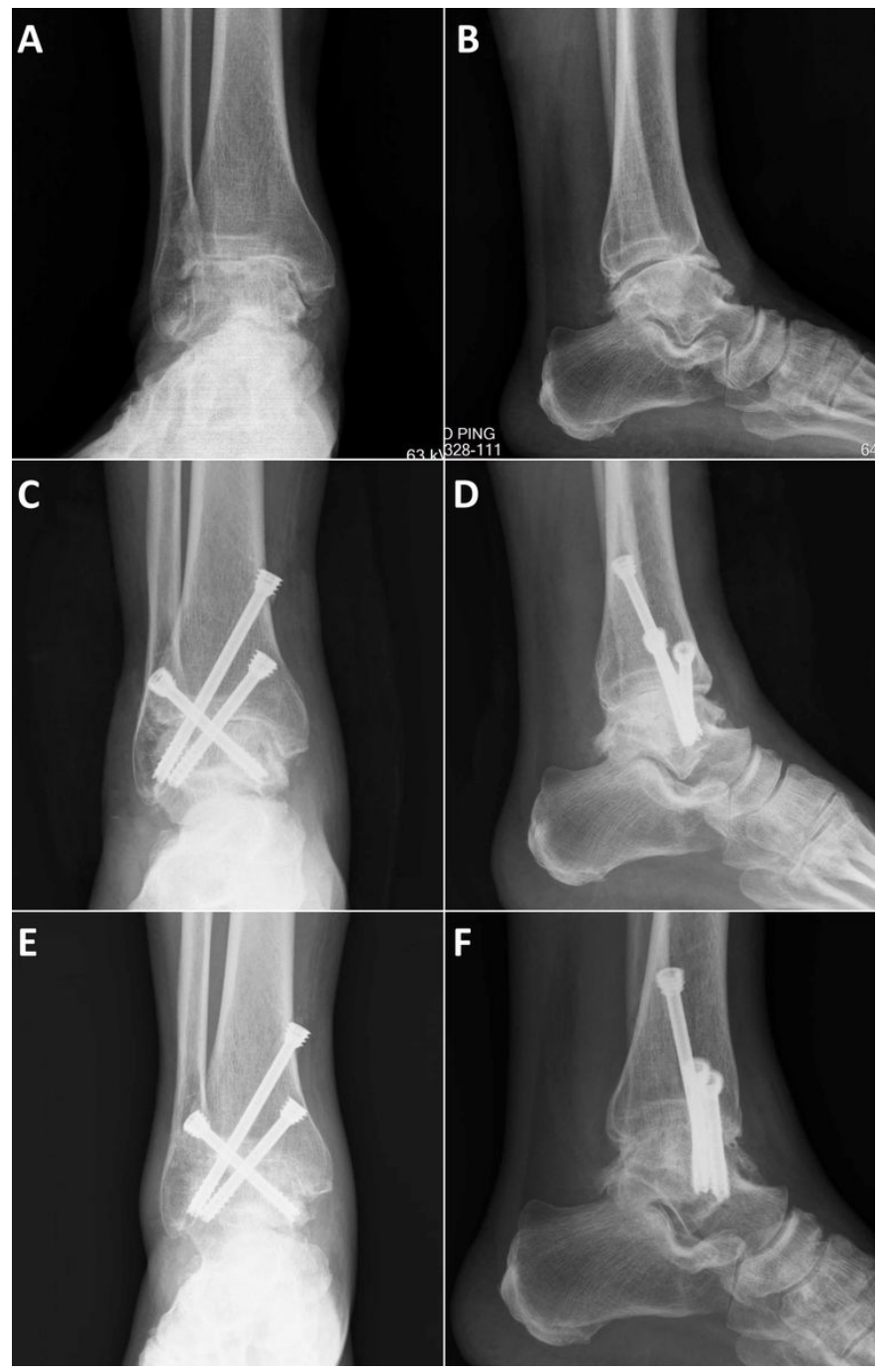

Figure 1

A typical case of AAF group, male, 63 years, the patient had a history of repeatedly sprained the right ankle for 8 years, and the ankle was found to be arthritis for 4 years: $(A, B)$ Anteroposterior and lateral X-ray film 5 days before operation, we found that the orientation of ankle mortise can be acceptable. $(C, D)$ Anteroposterior and lateral X-ray film 2 days after operation, we made an arthroscopic ankle fusion by using three large cannulated screws; the joint space was filled and pressurized. (E,F) Anteroposterior and lateral X-ray film 3 months after operation, no obvious internal fixation loosening was observed, and a lot of bone bridges were found in the joint space, the ankle fusion was ideal. 


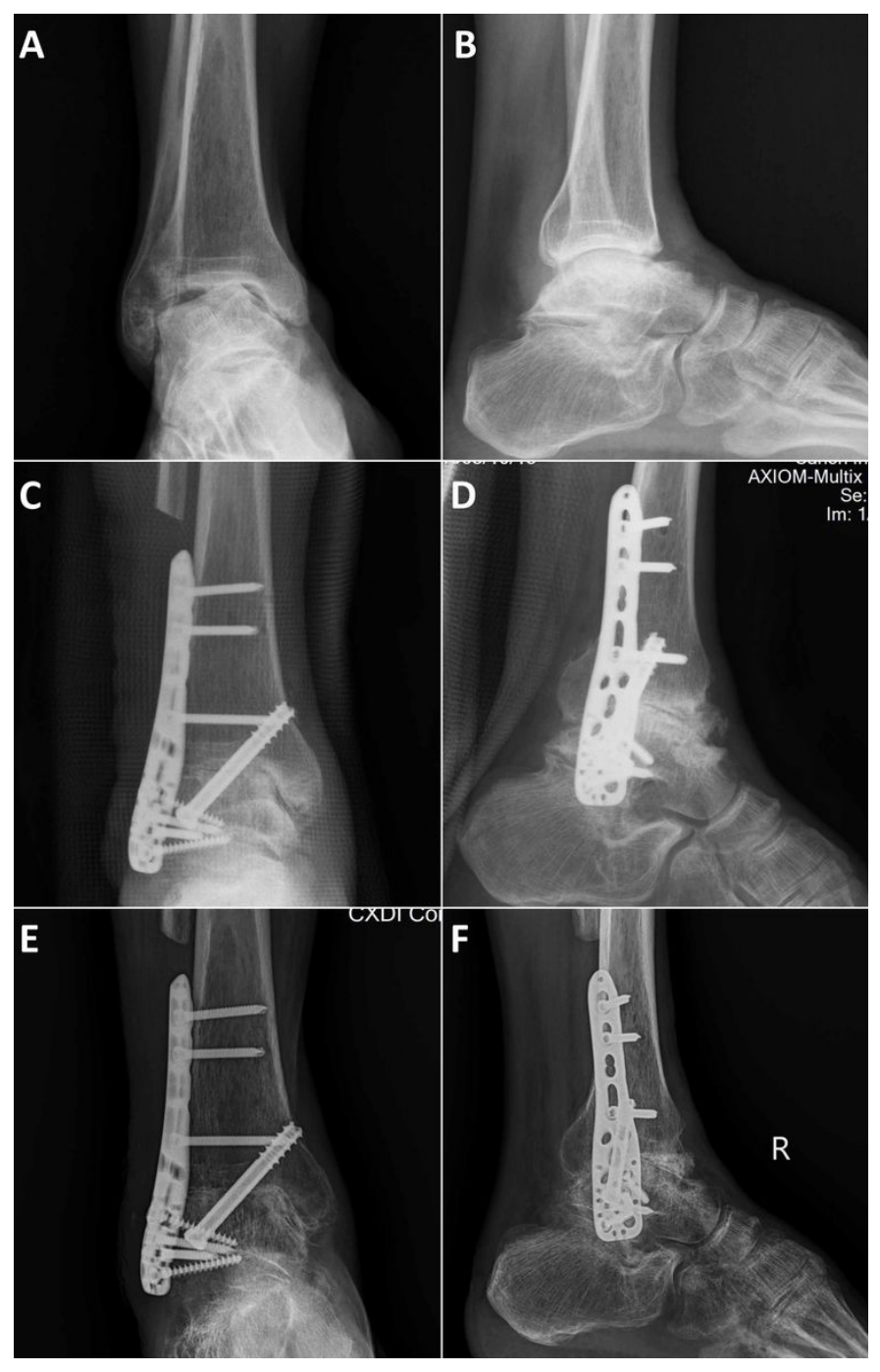

\section{Figure 2}

A typical case of SC subgroup, male, 49 years, the patient had a history of talus fractures 10 years ago, and followed by aseptic necrosis talus with ankle arthritis for 5 years: $(A, B)$ Anteroposterior and lateral X-ray film 3 days before operation, we can found that the talus was severely varus and collapsed, and was hardened with a lot of sequestrum. (C,D) Anteroposterior and lateral X-ray film 3 days after operation, we made distal fibular osteotomy and large lesion cleaning, the distal fibula was separated and crushed, for impacted and structural bone grafting, the joint space was filled and pressurized. (E,F) Anteroposterior and lateral X-ray film 3 months after operation, no obvious internal fixation loosening was observed, and a small number of bone bridges were found in the joint space; However, the ankle fusion was not ideal at this point, and the patient had been found a good fusion 6 months after operation. 


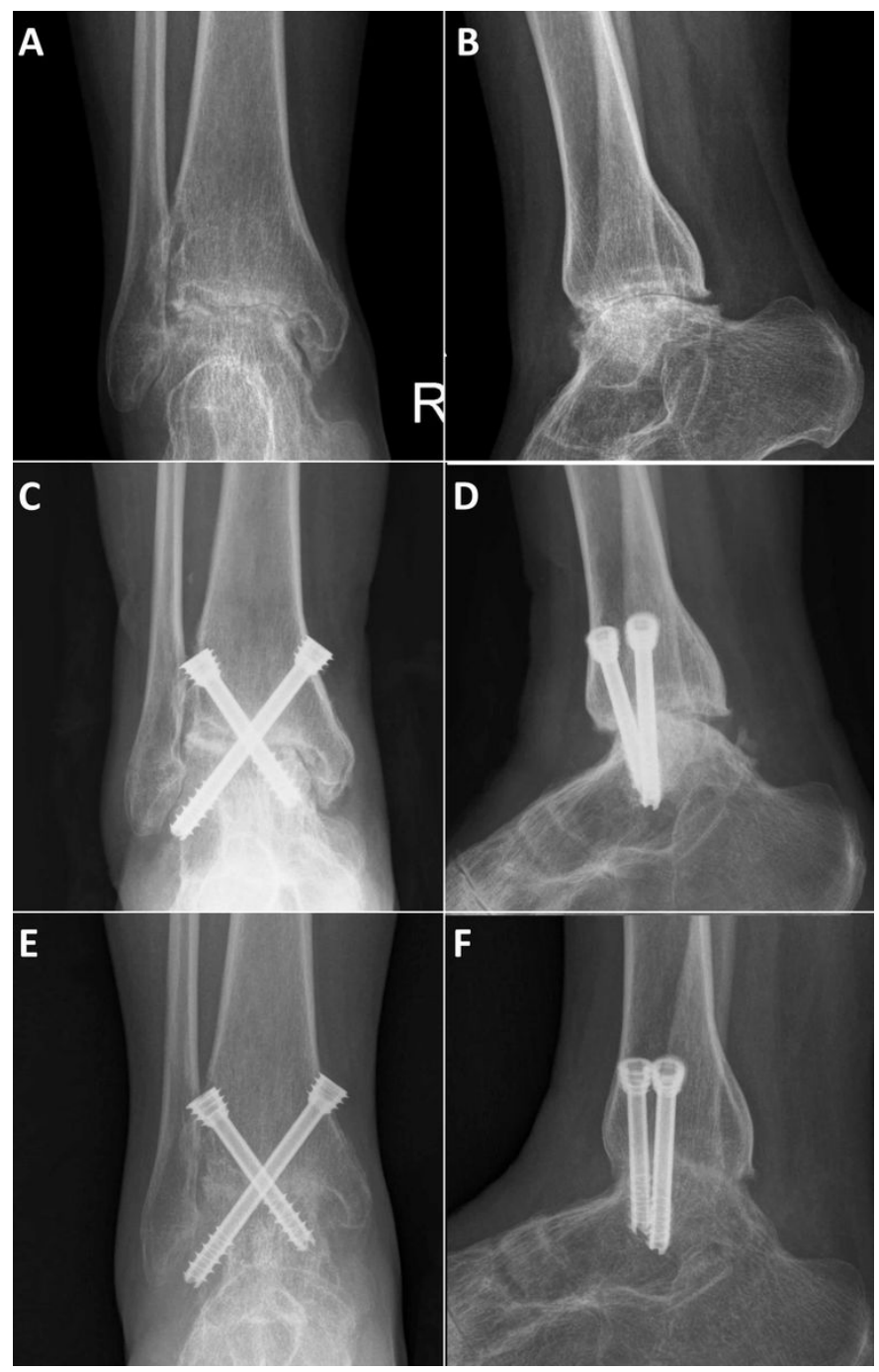

Figure 3

A typical case of NSC subgroup, female, 40 years, the ankle was found to be rheumatoid arthritis for 10 years: (A,B) Anteroposterior and lateral X-ray film 4 days before operation, we found that no obvious distortion of the ankle mortise orientation, but the joint space has disappeared and osteoporosis can be found. (C,D) Anteroposterior and lateral X-ray film 2 days after operation, we made an ankle fusion with an anterior approach by using two large cannulated screws; the joint space was pressurized. (E,F) Anteroposterior and lateral X-ray film 3 months after operation, no obvious internal fixation loosening was observed, and a lot of bone bridges were found in the joint space, the ankle fusion was ideal.

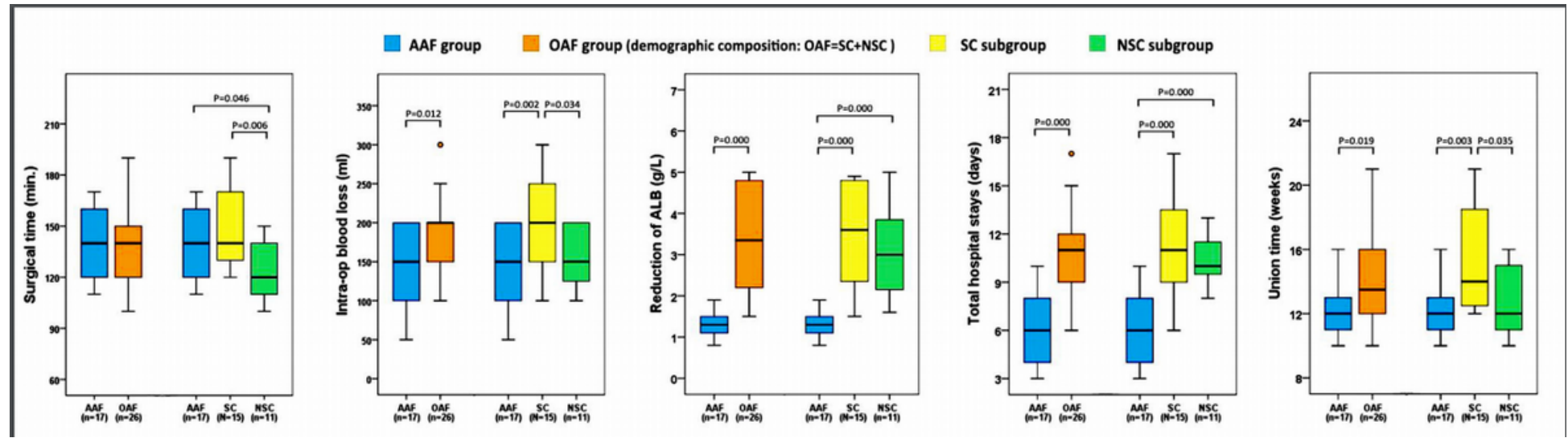

Figure 4

Box plot of surgical time, intra-op blood loss, reduction of ALB, total hospital stays and union time: We can found that the surgical time of NSC subgroup was significantly less than the AAF group and SC subgroup. The intra-op blood loss and the union time of AAF group were significantly less than the OAF 
group and the SC subgroup, and the NSC subgroup were significantly less than the SC subgroup too. The reduction of ALB and the total hospital stays of AAF group were significantly less than the OAF group, the SC subgroup and the NSC subgroup.

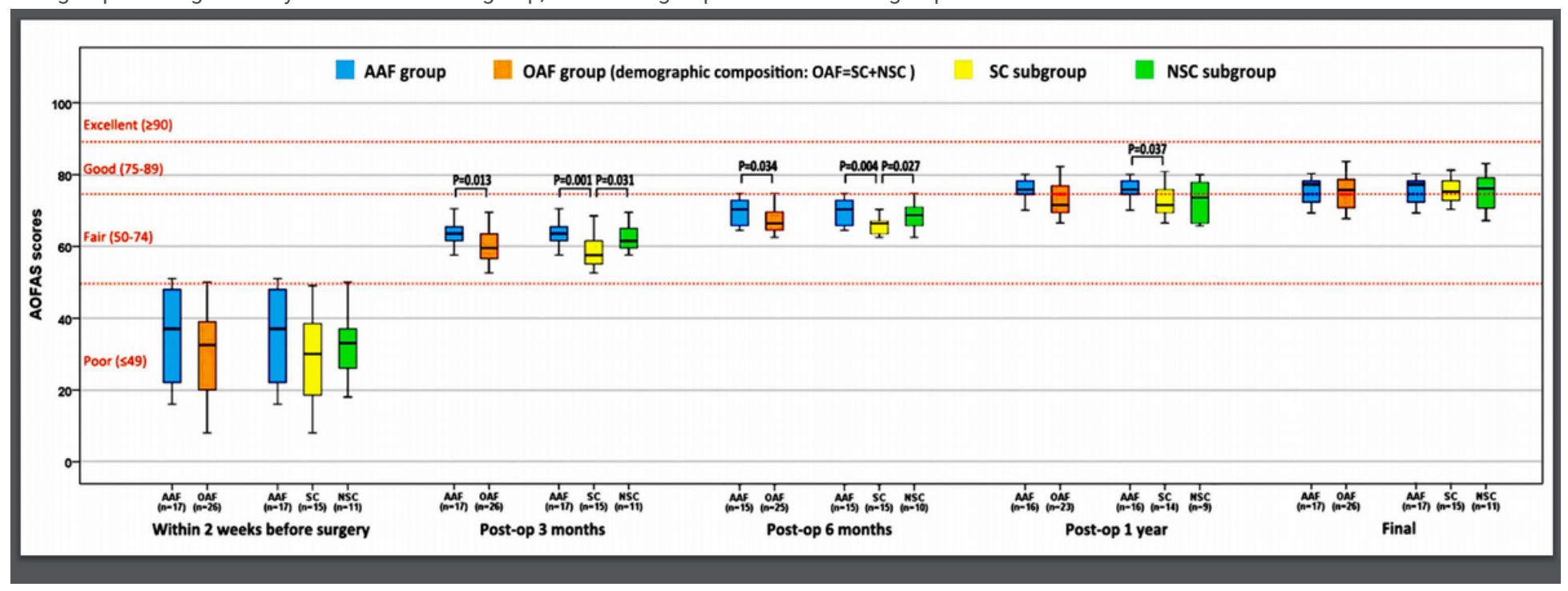

Figure 5

Box plot of AOFAS scores at every time points: The curative effect and recovery rate of AAF group and NSC subgroup were both better than the SC subgroup after operation until one year after operation; there was no significant difference between these three groups from one year after operation, and the AOFAS score mean of each group was greater than 75 points at that time point.

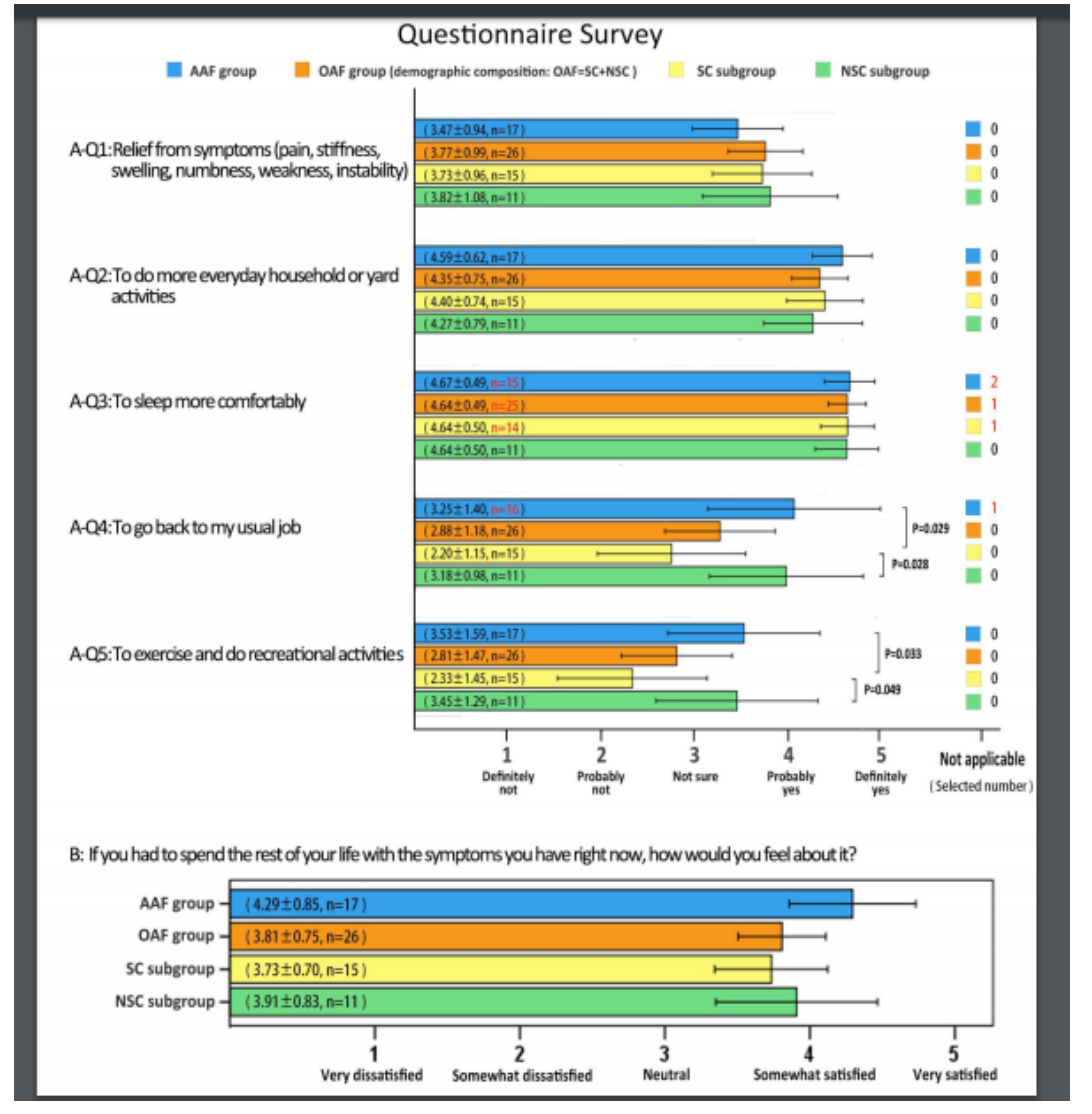

\section{Figure 6}

Outcomes of questionnaire survey: The survey was conducted during the final follow-up of the study. The questionnaire referred to Musculoskeletal Outcomes Data Evaluation and Management Scale (MODEMS) questionnaire, and each question was single choice. The case label of bar chart uses a 95\% confidence interval.

\section{Supplementary Files}


This is a list of supplementary files associated with this preprint. Click to download.

- Questionnaire.jpg

Page 16/16 